\title{
GRAIN SCALE CRYSTAL PLASTICITY MODEL WITH SLIP AND MICROTWINNING FOR A THIRD GENERATION NI-BASE DISK ALLOY
}

\author{
Jin E. Song ${ }^{1}$, David L. McDowell ${ }^{1,2}$ \\ ${ }^{1}$ George W. Woodruff School of Mechanical Engineering, Georgia Institute of Technology; Atlanta, GA 30332, USA \\ ${ }^{2}$ School of Materials Science \& Engineering, Georgia Institute of Technology; Atlanta, GA 30332, USA
}

Keywords: Ni-base disk superalloy, Crystal plasticity, Microtwinning, Constitutive model, ME3

\begin{abstract}
A relatively simple grain scale crystal plasticity model is developed for the third generation Ni-base disk superalloy ME3. The constitutive model embeds both microtwinning and octahedral and cube slip without explicitly modeling $\gamma^{\prime}$ precipitates and $\gamma$ matrix. The average size and volume fraction of $\gamma^{\prime}$ precipitates as well as the average grain size and crystallographic orientation are incorporated to embed sub-grain microstructural effects. Additionally, microtwinning is incorporated to specify the slip system shearing rate contribution via an Orowan equation, with kinetics based on diffusional reordering. The constitutive model is calibrated to straincontrolled cyclic histories and constant load creep experimental data for two supersolvus microstructures at $704^{\circ} \mathrm{C}$ and $760^{\circ} \mathrm{C}$
\end{abstract}

\section{Introduction}

Microstructure-sensitive constitutive relations for $\mathrm{Ni}$-base disk superalloys can be used to predict stress-strain-time responses for multiple purposes. For example, they enable the capability to modify the material to achieve targeted mechanical responses. Moreover, with the aid of improved computational power, it is becoming possible to implement elements of physically-based, fine scale constitutive models at the component level in aircraft gas turbine engines. This has been intractable in the past.

Microtwinning and slip are two potentially significant deformation mechanisms over the range of operating conditions of Ni-base gas turbine engine disk alloys. However, existing constitutive models address either microtwinning or slip, with the former dominant well below the initial yield point and the latter above. For relevance to a broad range of loading histories, a constitutive model should address both of these deformation mechanisms at the grain scale, with explicit polycrystal simulations above this scale or some scheme to inform macroscopic constitutive relations (cf. Shenoy et al. [1]).

In this work, such a constitutive model is informed by fine scale experiments and simulations, such as phase field modeling, and it is calibrated in a top-down manner using experimental responses of polycrystalline specimens subjected to various complex loading histories. The formulated crystal plasticity model can provide sensitivities to the underlying microstructure of Ni-base superalloy as well as to temperature and loading conditions such as strain rate and applied stress amplitudes.

\section{Deformation Mechanisms and Materials}

Ni-base superalloys are widely used in hot sections of aircraft gas turbine engines for their outstanding mechanical properties at elevated temperatures. Such improvements are mainly achieved by embedding high volume fractions of the ordered $\mathrm{L}_{2}$ fcc superlattice structured $\gamma^{\prime}$ particles in the fcc $\gamma$ matrix. The superalloy considered in this study is the third generation polycrystalline Ni-base disk superalloy ME3, the composition of which can be found in [2], with two supersolvus microstructures M1 and M2 considered, each containing bi-modal and tri-modal distribution of $\gamma^{\prime}$ precipitates, respectively. A range of microstructure attributes such as size and volume fractions of $\gamma^{\prime}$ precipitates as well as morphology and $\gamma$ matrix channel width between $\gamma^{\prime}$ particles are known to influence dislocation multiplication and migration. Depending on the microstructure attributes, particles can be either sheared or bypassed when encountered by dislocations that govern subsequent plastic deformation.

Recently, microtwinning has been found to be a significant independent deformation mechanism when Ni-base disk alloys are subjected to a constant stress (creep) at elevated temperature (approximately $650^{\circ} \mathrm{C}$ to $750^{\circ} \mathrm{C}$ ) and at stress levels below macroscopic yield. Strongly planar faults that form twins two to several tens of atomic planes thick (called microtwins) are found to traverse entire grains $[3,4,5,6]$. These microtwins serve as obstacles to slip or microtwinning on other slip (habit) planes and serve as the carriers for inelastic deformation under these conditions. Hence, the kinematics and kinetics of the two observed deformation mechanisms are implemented in the constitutive relation as appropriate.

\section{Constitutive Relation}

In this study, a constitutive relation for a grain scale crystal plasticity model is developed for a polycrystalline Ni-base disk superalloy, ME3. The constitutive model incorporates multiple deformation mechanisms observed under operating conditions of disk alloy applications. The crystal plasticity model assumes homogeneous anisotropic crystalline elasticity and plasticity within each grain without explicitly modeling $\gamma^{\prime}$ precipitates and $\gamma$ matrix. The model takes in average sizes and volume fractions of tri-modal $\gamma^{\prime}$ precipitate populations and embeds sub-grain microstructure effects. Furthermore, the average grain size and crystallographic orientation are directly implemented in the model with a total of 18 slip systems, including 12 octahedral and 6 cube slip systems, all of which are assumed to be active above the respective threshold stresses. The foundation of the model, absent microtwinning, is closely related to the work on IN100 by Shenoy [7], which is later explained in greater detail and accuracy in a comprehensive paper by Przybyla and McDowell [8].

The flow rule specifies the inelastic shearing rate on the $\alpha^{\text {th }}$ slip system using the resolved shear stress and the internal state 
variable (ISV) evolution. The inelastic shearing rate is composed of two additive contributions, i.e.,

$$
\dot{\gamma}^{\alpha}=\left[\dot{\gamma}_{1} \exp \left(-\frac{\mathrm{Q}_{1}}{\mathrm{k}_{\mathrm{B}} \mathrm{T}}\right)\left\langle\frac{\left|\tau^{\alpha}-\chi^{\alpha}\right|-\kappa_{\lambda}^{\alpha}}{\mathrm{D}_{\lambda}^{\alpha}}\right\rangle^{\mathrm{n}_{1}}\right] \operatorname{sgn}\left(\tau^{\alpha}-\chi^{\alpha}\right)+\dot{\gamma}_{\mathrm{mt}}^{\alpha}
$$

The first term (within the square brackets) is classical and represents the slip system shearing rate describing collective deformation by dislocation glide on octahedral and cube slip systems, where $\dot{\gamma}_{1}$ is the reference shearing rate, $\tau^{\alpha}$ is the resolved shear stress on $\alpha^{\text {th }}$ slip system, and $\chi^{\alpha}$ and $\mathrm{D}_{\lambda}^{\alpha}$ are the slip system back stress and the drag stress, respectively, with $\lambda$ standing for either the octahedral or the cube slip systems. Also, $\kappa_{\lambda}^{\alpha}$ is the microstructure-sensitive threshold slip resistance. The Arrhenius term includes Boltzmann's constant $\mathrm{k}_{\mathrm{B}}$, temperature in Kelvin, $\mathrm{T}$, with $\mathrm{Q}_{1}$ the activation energy for dislocation bypass. The Macauley bracket function is defined by $\langle\mathrm{g}\rangle=\mathrm{g} \mathrm{u}(\mathrm{g})$, such that it is nonzero only for positive values of the argument $\mathrm{g}$.

The second term represents the microtwinning-induced deformation to specify the slip system shearing rate contribution. The inelastic shearing rate is defined only on octahedral slip systems via an Orowan equation based on the kinetics of microtwin growth. The nucleation of microtwins is governed by dislocation dissociation which is observed to depend strongly on the magnitude and orientation of the resolved shear stress relative the leading and trailing partial dislocations, according to phase field model simulations conducted at The Ohio State University $[5,9,10]$.

The evolution equations of the ISVs are physically associated with deformation mechanisms observed under loading conditions of interest. Detailed descriptions of state variables and associated evolution equations are presented in the following sections.

\section{Threshold Slip Resistance and Non-Schmid Effects}

The threshold slip resistance is analogous to the yield strength; it serves as a threshold for slip at the slip system level. The threshold slip resistance depends on the microstructure attributes and reflects hardening behavior induced by slip plane obstacles and dislocation-dislocation interactions. The presentation of the model here follows that of Przybyla and McDowell [8], based on earlier developments of Shenoy [7]. The initial critical resolved shear stress (CRSS) is microstructure-sensitive and is determined for octahedral and cube slip systems by

$$
\begin{aligned}
& \kappa_{\mathrm{o}, \mathrm{oct}}^{\alpha}=\left[\tau_{\mathrm{o}, \mathrm{oct}}^{\alpha}+\mathrm{c}_{\mathrm{p} 2 \mathrm{~L}} \sqrt{\xi \frac{\mathrm{f}_{\mathrm{p} 2 \mathrm{~L}}^{\prime}}{\mathrm{d}_{\mathrm{p} 2 \mathrm{~L}}}}+\mathrm{c}_{\mathrm{p} 2 \mathrm{~S}} \sqrt{\xi \frac{\mathrm{f}_{\mathrm{p} 2 \mathrm{~S}}^{\prime}}{\mathrm{d}_{\mathrm{p} 2 \mathrm{~S}}}}+\mathrm{c}_{\mathrm{p} 3} \sqrt{\xi \mathrm{f}_{\mathrm{p} 3}^{\prime} \mathrm{d}_{3}}+\frac{\mathrm{c}_{\mathrm{gr}}}{\sqrt{\mathrm{d}_{\mathrm{gr}}}}\right] \\
& +\left(\mathrm{f}_{\mathrm{p} 2 \mathrm{~L}}+\mathrm{f}_{\mathrm{p} 2 \mathrm{~S}}\right) \tau_{\mathrm{ns}}^{\alpha} \\
& \kappa_{\mathrm{o}, \text { cub }}^{\alpha}=\left[\tau_{\mathrm{o}, \mathrm{cub}}^{\alpha}+\mathrm{c}_{\mathrm{p} 2 \mathrm{~L}} \sqrt{\xi \frac{\mathrm{f}_{\mathrm{p} 2 \mathrm{~L}}^{\prime}}{\mathrm{d}_{\mathrm{p} 2 \mathrm{~L}}}}+\mathrm{c}_{\mathrm{p} 2 \mathrm{~S}} \sqrt{\xi \frac{\mathrm{f}_{\mathrm{p} 2 \mathrm{~S}}^{\prime}}{\mathrm{d}_{\mathrm{p} 2 \mathrm{~S}}}}+\mathrm{c}_{\mathrm{p} 3} \sqrt{\xi \mathrm{f}_{\mathrm{p} 3}^{\prime} \mathrm{d}_{3}}+\frac{\mathrm{c}_{\mathrm{gr}}}{\sqrt{\mathrm{d}_{\mathrm{gr}}}}\right] \\
& \text { where } \xi=\frac{\Gamma_{\mathrm{APB}}}{\Gamma_{\mathrm{APB} \_ \text {ref }}}
\end{aligned}
$$

and $\Gamma_{\mathrm{APB}}$ is the anti-phase boundary (APB) energy. Here, coefficients $c *$ are material fitting parameters, and $\mathrm{f}_{\mathrm{p} 2 \mathrm{~L}}$ and $\mathrm{d}_{\mathrm{p} 2 \mathrm{~L}}$ are the respective volume fraction and size of large secondary $\gamma^{\prime}$ particles. Subscripts p2L, p2S, and p3 indicate large secondary, small secondary and tertiary $\gamma^{\prime}$ particles, respectively. The secondary $\gamma^{\prime}$ particles demonstrate a bimodal distribution, therefore are classified as 'large' and 'small' secondary $\gamma$ ' particles. Furthermore, normalized precipitate volume fractions are defined by

$$
f_{p 2 L}^{\prime}=\frac{f_{p 2 L}}{f_{p 2 L}+f_{m}}, f_{p 2 S}^{\prime}=\frac{f_{p 2 S}}{f_{p 2 S}+f_{m}}, f_{p 3 L}^{\prime}=\frac{f_{p 3 L}}{f_{p 3 L}+f_{m}}
$$

where $\mathrm{f}_{\mathrm{m}}$ is the austenitic $\gamma$ matrix volume fraction.

The non-Schmid stress $\tau_{\text {ns }}^{\alpha}$ is only applicable to the octahedral slip systems within $\gamma^{\prime}$ particles and governs the transformation from glissile to sessile dislocation cores via dislocation dissociation by implementing the Paidar-Pope-Vitek (PPV) model. The nonSchmid stress is defined as [11]

$$
\tau_{\mathrm{ns}}^{\alpha}=\mathrm{h}_{\mathrm{pe}} \tau_{\mathrm{pe}}^{\alpha}+\mathrm{h}_{\mathrm{se}} \tau_{\mathrm{se}}^{\alpha}+\mathrm{h}_{\mathrm{cb}}\left|\tau_{\mathrm{cb}}^{\alpha}\right|
$$

Here, $\tau_{\mathrm{pe}}^{\alpha}, \tau_{\mathrm{se}}^{\alpha}, \tau_{\mathrm{cb}}^{\alpha}$ are the resolved shear stresses on the primary, secondary and cube slip systems, and $\mathrm{h}_{\mathrm{pe}}, \mathrm{h}_{\mathrm{se}}, \mathrm{h}_{\mathrm{cb}}$ are corresponding temperature dependent coefficients. According to the PPV model [12], screw dislocations on (111) planes can be dissociated into two super-Shockley partials by APB bands. The configurations of the screw dislocations can be divided into: (1) glissile with its core in the same plane and (2) sessile with a non-planar core in the $(1 \overline{1} 1)$ plane which increases the threshold slip resistance.

The shear stresses on the primary and the cube slip systems of Eq. (4) manifest tension/compression asymmetry. The sign of the shear stress on the primary slip system, $\tau_{\mathrm{pe}}^{\alpha}$, determines whether the constriction or separation of the Shockley partial dislocations will occur; this in turn determines the possibility of cross-slip from the octahedral plane to the cube plane. The magnitude of the resolved shear stress on the cube slip system, $\tau_{\mathrm{cb}}^{\alpha}$, on the other hand, determines whether the cross slip will occur; this is independent of the direction of the resolved shear stress. As suggested in the PPV model, the influence of the shear stress on the secondary slip system, $\tau_{\mathrm{se}}^{\alpha}$, is included in the model.

For the total threshold slip resistance, the initial CRSS is added to a Taylor hardening term which describes the evolution of the threshold stress with dislocation density evolution. The overall slip resistance for octahedral and cube slip systems are written as

$$
\kappa_{\lambda}^{\alpha}=\kappa_{\mathrm{o}, \lambda}^{\alpha}+\left\langle\alpha_{\mathrm{t}} \mu_{\text {mix }} b_{\text {mix }} \sqrt{\rho_{\lambda}^{\alpha}}-\mathrm{C} \max \left(\left|\chi_{\lambda}^{\alpha}\right|\right)\right\rangle \text { for } \lambda=\text { oct, cub }
$$

where $\alpha_{\mathrm{t}}$ and $\mathrm{C}$ are fitting parameters, and $\rho_{\lambda}^{\alpha}$ is the dislocation density for the $\alpha^{\text {th }}$ slip system, as will be discussed further in the following section. Additionally, $\mu_{\text {mix }}$ and $b_{\text {mix }}$ are the volume averaged shear modulus and Burgers vector, respectively, i.e.,

$$
\begin{aligned}
& \mu_{\text {mix }}=\left(\mathrm{f}_{\mathrm{p} 2 \mathrm{~L}}+\mathrm{f}_{\mathrm{p} 2 \mathrm{~S}}+\mathrm{f}_{\mathrm{p} 3}\right) \mu_{\gamma^{\prime}}+\mathrm{f}_{\mathrm{m}} \mu_{\mathrm{m}} \\
& \mathrm{b}_{\text {mix }}=\left(\mathrm{f}_{\mathrm{p} 2 \mathrm{~L}}+\mathrm{f}_{\mathrm{p} 2 \mathrm{~S}}+\mathrm{f}_{\mathrm{p} 3}\right) \mathrm{b}_{\gamma^{\prime}}+\mathrm{f}_{\mathrm{m}} \mathrm{b}_{\mathrm{m}}
\end{aligned}
$$

where $\mu_{\gamma^{\prime}}$ and $\mu_{\mathrm{m}}$ are the shear moduli of the $\gamma^{\prime}$ precipitate and $\gamma$ matrix phases, and $b_{\gamma^{\prime}}$ and $b_{m}$ are the magnitudes of the Burgers vectors for $\gamma^{\prime}$ precipitates and $\gamma$ matrix, respectively. 
Experimentally, the isotropic slip resistance is observed to decrease at increasing inelastic strain magnitudes, while the back stress magnitude increases significantly. Specifically, the increase of back stress (kinematic hardening) is balanced by a decrease in the threshold stress (isotropic hardening). In other words, short range back stress is effectively indiscernible from isotropic strength under monotonic loading conditions, but it is evident in its effects upon cyclic loading which involves reversed yielding. This reflects the role of complex dislocation-precipitate interactions that are shorter ranged in character than intergranular interactions. It is presumed that the initial threshold resistance is high because $\gamma^{\prime}$ precipitates are not yet cut, but they become easier to pass through as the straining continues. This reduces the nondirectional slip resistance while increasing the back stress as cycling continues and inelastic strain accumulates. Therefore, $\gamma^{\prime}$ precipitates become easier to shear but with higher directionality in slip system dislocation network resistance. Similar phenomena have been previously observed [13].

\section{Dislocation Density}

In the crystal plasticity model, the dislocation density on each slip system plays an important role in driving the evolution of the ISVs. The hardening behavior is caused by the multiplication and interaction of dislocations with barriers established by statistically stored dislocations (SSDs) and geometrically necessary dislocations (GNDs) associated with dislocation pileups at shearable precipitates (dominant at high $\gamma^{\prime}$ particle volume fractions) at the scale of precipitate spacing. Dislocation multiplication and recovery are considered [7], as well as dislocation annihilation [14]. The dislocation density evolution in the $\gamma$ matrix expresses the competition between storage and annihilation of dislocations, with the dislocation density evolution written as [14]

$$
\dot{\rho}_{\lambda}^{\alpha}=\mathrm{h}_{\lambda}^{\alpha \alpha}\left\{\mathrm{Z}_{0}+\mathrm{k}_{1} \sqrt{\rho_{\lambda}^{\alpha}}-\mathrm{k}_{2} \rho_{\lambda}^{\alpha}\right\}\left|\dot{\gamma}_{\lambda}^{\alpha}\right|-\mathrm{C}_{\rho \mathrm{R}}\left(\rho_{\lambda}^{\alpha}\right)^{m_{R}}
$$

where $\mathrm{k}_{1}$ and $\mathrm{k}_{2}$ are material constants, and $\mathrm{Z}_{0}$ accounts for particle spacing via the relations

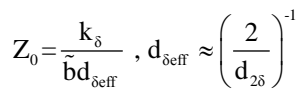

Here, $\mathrm{k}_{\delta}$ is a constant, and $\mathrm{d}_{2 \delta}$ is the spacing between secondary $\gamma^{\prime}$ precipitates. The model assumes only self-hardening. Also, the effective precipitate spacing, $\mathrm{d}_{\delta \mathrm{eff}}$, is calculated based on both large and small secondary $\gamma^{\prime}$ precipitate spacings. The term $Z_{0}$ models geometrically necessary dislocation (GND) density evolution [7]. Furthermore, a diffusional recovery term (far right in Eq. 7) is employed to model the reduction in dislocation density via static thermal recovery (diffusional rearrangement and annihilation).

\section{Back Stress Evolution}

The kinematic hardening is intended to capture the Bauschinger effect [15]. The slip system back stress evolution in the crystal plasticity model is given by

$$
\dot{\chi}_{\lambda}^{\alpha}=C_{\chi}\left\{\eta_{o_{0}} \mu_{\gamma^{\prime}} \tilde{b} \sqrt{\rho_{\lambda}^{\alpha}} \operatorname{sgn}\left(\tau^{\alpha}-\chi_{\lambda}^{\alpha}\right)-\chi_{\lambda}^{\alpha}\right\}\left|\dot{\gamma}^{\alpha}\right|-\beta\left(\chi_{\lambda}^{\alpha}\right)^{2} \operatorname{sgn}\left(\chi_{\lambda}^{\alpha}\right)
$$

where $C_{\chi}$ is a fitting parameter for evolution rate, and $\eta_{o}$ is a coefficient that determines the amplitude of the back stress. The static thermal recovery term (last term in Eq. 9) captures the relaxation behavior during dwell periods as channel dislocations rearrange. The static thermal recovery coefficient $\beta$ is temperature dependent, i.e.,

$$
\beta=\mathrm{A}_{2} \exp \left(\frac{\mathrm{Q}_{\text {self }}}{\mathrm{RT}}\right)^{\mathrm{m}}
$$

where $\mathrm{A}_{2}$ and $\mathrm{m}$ are material constants and $\mathrm{Q}_{\text {self }}$ is the activation energy for self-diffusion in the $\gamma$ matrix.

\section{Microtwinning}

Microtwinning-induced deformation is incorporated as a contribution to the slip system shearing rate in the flow rule of Eq. (1) via an Orowan equation

$$
\dot{\gamma}_{\mathrm{mt}}^{\alpha}=\mathrm{b}_{\mathrm{tp}} \mathrm{v}^{\alpha} \rho_{\mathrm{mt}}^{\alpha}
$$

where $b_{\text {tp }}$ is the Burgers vector for the twinning partial dislocation, $\mathrm{v}^{\alpha}$ is the microtwin velocity, and $\rho_{\mathrm{mt}}^{\alpha}$ is the microtwin density on the $\alpha^{\text {th }}$ octahedral slip system.

According to recent studies on microtwinning $[4,5,6,9,16,17$, 18], the low stress creep deformation of Ni-base disk superalloys at elevated temperature is closely related to the mitigation of a/6<112> Shockley partial dislocations. Dissociation into leading and trailing partial dislocations is a necessary precursor to microtwinning. Dissociated partial dislocations are then reordered by a diffusion-mediated process and repeatedly shear through the $\gamma$ matrix and $\gamma^{\prime}$ precipitates, resulting in microtwin growth $[5,19]$.

Dislocation dissociation is implemented in the crystal plasticity model as a criterion for microtwin nucleation on each octahedral slip system. According to microscopic phase field model simulations conducted at the Ohio State University (Y. Wang, 2010), dislocation dissociation is observed to strongly depend on the magnitude and orientation of the resolved shear stress acting on the leading and trailing partial dislocations. Additionally, microstructure details such as $\gamma^{\prime}$ size, morphology and $\gamma$ matrix channel width are also assumed to significantly influence the dissociation process. Further information on dissociation mechanism can be found in [5].

Based on transmission electron microscopy (TEM) analysis (M. Mills, 2010) and the aforementioned phase field simulation results from work at The Ohio State University, cooperative dislocation behavior is approximated and dissociation criteria are established for microtwin nucleation and growth. The phase field model provides a range of the resolved shear stress magnitudes that can dissociate dislocations into partial dislocations. The upper limit of the range is defined by the resolved shear stress magnitude at which full dislocations appear in slip (constriction of partials); while the lower limit is defined by the resolved shear stress magnitude below which full dislocations are completely pinned at clusters of small $\gamma^{\prime}$ precipitates (i.e., they do not dissociate). These upper and lower limits, however, vary significantly depending on the orientation of the resolved shear stress. Therefore, dislocations on the corresponding slip system are observed to dissociate into the leading and trailing partials and form a microtwin when the 
resolved shear stress magnitude in the direction of the Burgers vector of the leading partial dislocation lies within a specified range. Once a microtwin is nucleated, as a simplification to accord with the observation of a dominant, highly planar microtwinned domain in each region of the crystal, the remaining slip systems are excluded from contributing to microtwin growth and the resulting shearing rate.

The resolved shear stress acting on the leading or trailing partial dislocation is calculated on each slip system to evaluate the dislocation dissociation criteria. The crystal plasticity model utilizes the $2^{\text {nd }}$ Piola-Kirchhoff stress, $\sigma_{\mathrm{pk} 2}$, in the intermediate configuration as the driving force for slip. The traction vector for slip system $\alpha$ acting on a slip plane in the intermediate configuration is given by

$$
\mathbf{T}^{\alpha}=\mathbf{n}_{\mathrm{o}}^{\alpha} \cdot \boldsymbol{\sigma}_{\mathrm{pk} 2}
$$

where $\mathbf{n}_{o}^{\alpha}$ is the unit vector normal to the slip plane containing slip system $\alpha$. The traction vector can be used to calculate the resolved shear stress $\tau_{\text {lead/trail }}^{\alpha}$ acting on either leading or trailing partial dislocation for slip system $\alpha$, i.e.,

$$
\tau_{\text {lead/trail }}^{\alpha}=\mathbf{T}^{\alpha} \cdot \mathbf{n}_{\text {lead/trail }}^{\alpha}
$$

where $\mathbf{n}_{\text {lead/trail }}^{\alpha}$ is a unit vector in the direction of the leading or trailing partial dislocation Burgers vector, as appropriate. Based on the argument set forth in the previous section, the dissociation criterion is stated as

$$
\kappa_{\text {mto }} \leq \max _{\alpha=1, \ldots, 12}\left(\tau_{\text {lead }}^{\alpha}\right) \leq \kappa_{\text {mtu }}
$$

where $\kappa_{\mathrm{mto}}$ and $\kappa_{\mathrm{mtu}}$ are the lower and upper dissociation threshold stresses, equivalent to the threshold stress bounds for microtwinning. For each microstructure considered in this study, $\kappa_{\text {mto }}$ and $\kappa_{\text {mtu }}$ are estimated based on phase field model simulations which account for microstructural details such as the $\gamma$ matrix channel width, the internal stacking fault energy, and the line tension of the associated microstructure.

This criterion for dislocation dissociation is implemented in an ABAQUS [20] User MATerial (UMAT) for crystal plasticity by computing the magnitudes of the resolved stress state acting on all 24 leading and trailing partial dislocations on octahedral planes at each integration point. When the maximum of the resolved shear stresses acting on all 12 leading partial dislocations exceeds the lower threshold stress for dislocation dissociation, the corresponding specific slip system is stored and triggers microtwin evolution. On the remaining slip systems, only dislocation glide is considered as an operative deformation mechanism (although it is unfavorable at comparably low resolved shear stresses). The microtwin-triggered slip system then drives microtwin evolution until the stress reaches the upper limit for dissociation.

\section{Microtwinning Kinetics}

Inelastic deformation via microtwinning was previously modeled with an Orowan equation for René 104 at $704^{\circ} \mathrm{C}$ at $838 \mathrm{MPa}$ [3].
A similar approach is taken with the microtwinning velocity on the active system, given by

$$
\begin{aligned}
& \mathrm{v}^{\alpha}=\frac{\mathrm{b}_{\mathrm{tp}}}{\mathrm{x}^{2}} \mathrm{D}_{\text {ord }}\left\{\ln \left(\frac{\left(\mathrm{f}_{\mathrm{p} 2 \mathrm{~L}}+\mathrm{f}_{\mathrm{p} 2 \mathrm{~S}}\right)\left(\Gamma_{\mathrm{pt}}-\Gamma_{\mathrm{tt}}\right)}{2\left(\tau_{\text {lead }}^{\alpha}\right) \mathrm{b}_{\mathrm{tp}}-\left(\mathrm{f}_{\mathrm{p} 2 \mathrm{~L}}+\mathrm{f}_{\mathrm{p} 2 \mathrm{~S}}\right) \Gamma_{\mathrm{tt}}}\right)\right\}^{-1} \operatorname{sgn}\left(\tau_{\text {lead }}^{\alpha}\right) \\
& \text { for } \kappa_{\mathrm{mtL}}<\tau_{\text {lead }}^{\alpha}<\kappa_{\mathrm{mtU}} \\
& \text { where } \\
& \kappa_{\mathrm{mtL}}=\min \left(\kappa_{\mathrm{mto}} \frac{\left(\mathrm{f}_{\mathrm{p} 2 \mathrm{~L}}+\mathrm{f}_{\mathrm{p} 2 \mathrm{~S}}\right) \Gamma_{\mathrm{tt}}}{2 \mathrm{~b}_{\mathrm{tp}}}\right), \kappa_{\mathrm{mtU}}=\max \left(\kappa_{\mathrm{mtu}}, \frac{\left(\mathrm{f}_{\mathrm{p} 2 \mathrm{~L}}+\mathrm{f}_{\mathrm{p} 2 \mathrm{~S}}\right) \Gamma_{\mathrm{pt}}}{2 \mathrm{~b}_{\mathrm{tp}}}\right)
\end{aligned}
$$

The relation is obtained from equating the energy decrease resulting from transforming a pseudo twin to a true twin with the work done by the resolved shear stress on the leading partial dislocation, $\tau_{\text {lead }}^{\alpha}$, in sweeping the microtwin through the matrix and secondary $\gamma^{\prime}$ precipitates. According to Kathikeyan et al. [3], $D_{\text {ord }}$ is the diffusion coefficient for reordering, and $\Gamma_{p t}, \Gamma_{t t}$ are pseudo and true twin activation energies, respectively. This relation is only valid when $\tau_{\text {lead }}^{\alpha}$ exceeds the secondary precipitate and true twin activation energy effect, $\left(f_{\mathrm{p} 2 L}+f_{\mathrm{p} 2 \mathrm{~S}} \Gamma_{\mathrm{tt}} / 2 \mathrm{~b}_{\mathrm{tp}}\right.$ (theoretical lower limit), and when $\tau_{\text {lead }}^{\alpha}$ is less than $\left(\mathrm{f}_{\mathrm{p} 2 \mathrm{~L}}+\mathrm{f}_{\mathrm{p} 2 \mathrm{~S})} \Gamma_{\mathrm{pt}} / 2 \mathrm{~b}_{\mathrm{tp}}\right.$, the theoretical upper limit set by athermal pseudo twin generation. Therefore, the lower threshold stress for microtwinning, $\kappa_{\mathrm{mtL}}$, is defined such that $\tau_{\text {lead }}^{\alpha}$ has to overcome both the lower dissociation threshold stress, $\kappa_{\text {mto }}$, and the theoretical lower limit to initiate microtwins. The upper threshold stress of microtwinning is also defined similarly.

\section{Microtwinning Density Evolution}

In the work of Karthikeyan et al. [3], the density of microtwins was assumed to be constant, based on the value obtained at the end of the creep test by TEM observations; this leads to an overestimation of the density in the early stages of deformation. In the present work, the microtwin density is formulated to evolve throughout the deformation process.

Microtwin density evolution can be formulated in terms of a heterogeneous nucleation model and subsequent growth. First, evolution of microtwin nucleation can be quantified by $\mathrm{n} / \mathrm{N}$, where $\mathrm{n}$ is the number of activated dissociation sites and $\mathrm{N}$ is the total number of potential dissociation sites. If $f\left(\tau_{\text {lead }}\right)$ is defined as the cumulative probability distribution of critical shear stress on the leading partial dislocation required for dissociation, the progression of microtwin nucleation can be estimated by integrating $f\left(\tau_{\text {lead }}\right)$ over the range of applied stress, i.e.,

$$
\int_{0}^{\tau_{\text {ked }}} f\left(\tau_{\text {lead }}\right) d \tau_{\text {lead }}=\left(\frac{\mathrm{n}}{\mathrm{N}}\right) \quad \text { for } \tau_{\text {lead }} \in\left(\kappa_{\mathrm{mtL}}, \kappa_{\mathrm{mtU}}\right]
$$

where $\kappa_{\mathrm{mtU}}$ and $\kappa_{\mathrm{mtL}}$ are upper and lower threshold stresses for microtwinning, as described previously in Eq. (14) and (15). The corresponding rate of the microtwin density $\rho_{\mathrm{mt}}^{\alpha}$ in a given volume $\mathrm{V}$ can be defined as

$$
\frac{d \rho_{\mathrm{mt}}^{\alpha}}{d \mathrm{t}}=\frac{\overline{1}}{\mathrm{~V}} \frac{d \mathrm{n}}{d \mathrm{t}}
$$


where $\overline{1}$ is the dislocation segment length generated by a dissociation event. By rearranging Eq. (17) and substituting into Eq. (16), the microtwin density evolution may be written for positive $\dot{\tau}_{\text {lead }}^{\alpha}$ as

$$
\dot{\rho}_{\mathrm{mt}}^{\alpha} \approx\left(\frac{\mathrm{N}}{\mathrm{V}} \bar{l}\right) f\left(\tau_{\text {lead }}^{\alpha}\right) \dot{\tau}_{\text {lead }}^{\alpha}
$$

Here, $\overline{1}$ can be assumed to be equivalent to the critical size of $\gamma^{\prime}$ precipitates $d_{d s}$ which promotes dislocation dissociation and is tentatively determined by phase field model simulations. The number of potential dislocation dissociation sites in a given volume is defined as

$$
\frac{\mathrm{N}}{\mathrm{V}}=\frac{\left(\mathrm{f}_{\mathrm{uf}}+\alpha^{3} \mathrm{f}_{\mathrm{p} 3}\right)}{\left(\mathrm{d}_{\mathrm{ds}}+\mathrm{w}\right)^{3}}
$$

where $f_{u f}$ is the volume fraction of secondary $\gamma^{\prime}$ precipitates (both large and small), $w$ is the channel width between $\gamma^{\prime}$ particles, and $\alpha$ is an effective volume fraction factor for tertiary $\gamma^{\prime}$ particles.

It is assumed that some tertiary $\gamma^{\prime}$ particles cluster and behave in a collective manner, similar to the behavior of secondary $\gamma^{\prime}$ particles, by dissociating dislocations that encounter them. Therefore, the volume fraction of tertiary $\gamma^{\prime}$ precipitates is factored in Eq. (19) and contributes to potential microtwin nucleation sites. Within a cluster of tertiary $\gamma^{\prime}$ particles, however, narrow $\gamma$ matrix channels exist between tertiary $\gamma^{\prime}$ particles, and the volume fraction of tertiary $\gamma^{\prime}$ precipitates $\mathrm{f}_{\mathrm{p} 3}$ is not a reasonable representation of the volume occupied by the clusters. Hence, the effective volume fraction factor $\alpha^{3}$ is multiplied by the volume fraction of tertiary $\gamma^{\prime}$ particles so that the space between tertiary $\gamma^{\prime}$ particles can also be taken into account. Eq. (19) can be substituted into Eq. (18), giving the microtwin density rate

$$
\dot{\rho}_{\mathrm{mt}}^{\alpha}=\left[\frac{\mathrm{f}_{\mathrm{uf}}+\alpha^{3} \mathrm{f}_{\mathrm{p} 3}}{\left(\mathrm{~d}_{\mathrm{ds}}+\mathrm{w}\right)^{3}}\right] \mathrm{d}_{\mathrm{ds}} f\left(\tau_{\text {lead }}^{\alpha}\right) \dot{\tau}_{\text {lead }}^{\alpha}
$$

It is noted that nucleation is not considered rate sensitive in this formulation, in contrast to growth (cf. Eqs. (11) and (15) together). The cumulative probability density function $f\left(\tau_{\text {lead }}\right)$ is selected to be of the form

$$
f\left(\tau_{\text {lead }}^{\alpha}\right)=\left(\frac{\tau_{\text {lead }}^{\alpha}-\kappa_{\text {mtL }}}{\kappa_{\text {mtU }}-\kappa_{\text {mtL }}}\right)^{m}, \quad \text { for } \kappa_{\text {mtL }}<\tau_{\text {lead }}^{\alpha}<\kappa_{\text {mtU }}
$$

where $\mathrm{m}$ is a parameter that controls the shape of the probability distribution function. The microtwin density evolution can be rewritten by introducing Eq. (21) to Eq. (18), i.e.,

$$
\dot{\rho}_{\mathrm{mt}}^{\alpha}= \begin{cases}{\left[\frac{\mathrm{f}_{\mathrm{uf}}+\alpha^{3} \mathrm{f}_{\mathrm{p} 3}}{\left(\mathrm{~d}_{\mathrm{ds}}+\mathrm{w}\right)^{3}}\right]\left(\frac{\tau_{\text {lead }}^{\alpha}-\kappa_{\mathrm{mtL}}}{\kappa_{\mathrm{mtU}}-\kappa_{\mathrm{mtL}}}\right)^{m} \mathrm{~d}_{\mathrm{ds}} \dot{\tau}_{\text {lead }}^{\alpha}} & \text {, for } \kappa_{\mathrm{mtL}} \leq \tau_{\text {lead }}^{\alpha} \leq \kappa_{\mathrm{mtU}} \\ 0, \text { otherwise } & \end{cases}
$$

The formulated microtwin density evolution shows a low rate of evolution in the early stage of microtwinning followed by a rapid increase.

It should also be noted that the model has certain elements of uncertainty. For example, in Eq. (22), the effective $\gamma^{\prime}$ particle size $\mathrm{d}_{\mathrm{ds}}$ and spacing $\mathrm{w}$ are estimated based on microstructure attributes without measuring the actual distribution or conducting detailed analysis. The upper and lower threshold stresses $\kappa_{\mathrm{mtu}}$ and $\kappa_{\mathrm{mto}}$ are also estimated from phase field model simulations based on the average spacing $\mathrm{w}$ and are subject to approximations made by that model. Moreover, various model forms (e.g., PDF in Eq. (21)) and model parameters have epistemic uncertainty that can be reduced with more experimental results, for example.

\section{Model Implementation}

The constitutive relation for the crystal plasticity model is implemented as an ABAQUS UMAT [20]. Within the UMAT, the inelastic shearing rate is calculated for each slip system using a fully implicit integration scheme with incremental line search and a Newton-Raphson technique. Additionally, time step subincrementation prevents the inelastic shearing rate from diverging by sub-incrementing the global time step. Further description can be found in the dissertation of McGinty [21].

\section{Validation and Calibration}

The grain scale microstructure-sensitive crystal plasticity model is further refined and calibrated based on various experimental responses under a range of loading conditions. Two types of experiments are employed: 24 strain-controlled and 11 stresscontrolled loads, applied to two supersolvus microstructures. Strain-controlled experiments involve strain histories with various strain amplitudes accompanied by dwell periods and are conducted at strain rates of $10^{-3} \mathrm{~s}^{-1}$ and $10^{-5} \mathrm{~s}^{-1}$ at $704^{\circ} \mathrm{C}$ and $760^{\circ} \mathrm{C}$. Experimental responses generated with strain-controlled test conditions are used to calibrate parameters associated with dislocation glide. Stress-controlled creep responses at multiple stress levels are used to estimate parameters of the microtwinning relations.

Calibration of the crystal plasticity model is not automated for several reasons. First, polycrystal plasticity simulations are computationally intensive and are therefore not amenable to the myriad of runs that are necessary to support automated iterative optimization. Efficient metamodels that are able to explore microstructure sensitivity as a replacement for polycrystal plasticity models are not available. Second, such a model framework is highly nonlinear with many local minima of an objective function based on global responses; accordingly, attempts at automation are fraught with challenges in searching sub-optimal regions even when considering constraints on physically-based parameters such as activation energies, elastic constants, and so forth. Instead, these are specified and clusters of parameters are iteratively optimized. Hence, a manual calibration/update process aided by optimization in targeted domains remains as a practical necessity for the crystal plasticity model.

The calibration process involves multiple aspects to be considered simultaneously; it can be divided into four steps/aspects: (1) 
mechanical behavior, (2) strain rate effects, (3) temperature effects, and (4) microstructure effects.

The first step considers calibration with respect to measured mechanical behavior. Initially, only the low strain range of experimental responses is used to analyze the yield point and early post yield behavior, and some of the most influential constants in terms of elastic and plastic behaviors in this regime are estimated. Then, consideration of response is extended to include higher levels of plastic deformation. At this stage, constants associated with the dislocation density, back stress, and slip resistance threshold stress are calibrated to capture the overall shapes of stress-strain relation at substantial plastic deformation. In general, parameters are estimated based on compromise fitting of various responses. This calibration procedure is conducted separately using individual experiments, and relaxation behaviors during dwell periods are initially neglected.

The next calibration step is focused on the relaxation response during strain hold periods; the static thermal recovery constants in the dislocation density and back stress evolution equations are calibrated to dwell responses of individual experiments at this stage. Increasing the influence of static thermal recovery terms may shift the stress-strain hysteresis slightly; therefore previously calibrated parameters need to be adjusted accordingly. At this point, there should be multiple sets of constants calibrated to the relatively simple experimental datasets considered. The calibrated set of parameters from each experiment is then applied to experiments with complex loading histories and readjusted to improve fitting. In general, this step introduces a slight compromise of fitting among multiple experiments; however, the focus should be placed more on capturing mechanical behaviors such as elastic and inelastic deformation as well as relaxation behaviors over the range of considered loading conditions.

The next aspect to consider is the strain rate sensitivity. The stress amplitude is observed to drop significantly when the strain rate is decreased from $10^{-3} \mathrm{~s}^{-1}$ to $10^{-5} \mathrm{~s}^{-1}$. Consequently, model parameter sets calibrated at different strain rates are compared, and the flow rule exponent and the drag stress are adjusted to capture the decrease in stress. A similar strategy is employed for the temperature effect where a significant stress decrease is observed when temperature is increased from $706^{\circ} \mathrm{C}$ to $760^{\circ} \mathrm{C}$. The crystal plasticity model is formulated with temperature influence through physically based means, including thermally activated processes via Arrhenius-type relations, as listed in Table I. As in the rate sensitivity case, time-stress-strain responses at both temperatures are monitored while constants associated with temperature are adjusted to find a parameter set that can adequately predict responses at both temperatures. Calibrated parameters with temperature dependence are listed in Table II.
Table I. Temperature dependence of crystal plasticity model parameters in the range of $706^{\circ} \mathrm{C}$ to $760^{\circ} \mathrm{C}$

\begin{tabular}{|c|c|}
\hline $\begin{array}{l}\text { Set 1. Initial CRSS } \\
\mathrm{c}_{\mathrm{p} i}=\left.\frac{\mu_{i}}{\mu_{\mathrm{T}=650^{\circ} \mathrm{C}}} \tilde{\mathrm{c}}_{\mathrm{p} i}\right|_{\mathrm{T}=650^{\circ} \mathrm{C}} \\
\text { where } i=2 \mathrm{~L}, 2 \mathrm{~S}, 3 \\
\mathrm{R}=8.314 \mathrm{~J} / \mathrm{mol}-\mathrm{K}\end{array}$ & $\begin{array}{l}\text { Set 2. Non-Schmid (PPV) } \\
\mathrm{h}_{\mathrm{pe}}=2 \cdot \mathrm{h}_{\mathrm{se}}=\mathrm{A} \exp \left(\frac{\mathrm{Q}_{c s}}{\mathrm{RT}}\right)^{p} \\
\text { where } \mathrm{Q}_{c s}=170 \mathrm{~kJ} / \mathrm{mol} \\
p=0.52 \\
\mathrm{~A}=8.02 \times 10^{-6}\end{array}$ \\
\hline $\begin{array}{l}\text { Set 3. Dislocation Density } \\
\text { Dynamic Recovery } \\
\mathrm{k}_{2}=\mathrm{k}_{2 \mathrm{ref}} \exp \left(-\frac{\mathrm{Q}_{c s}}{\mathrm{RT}}\right)^{b} \\
\text { where } \mathrm{k}_{2 \mathrm{ref}}=1.13 \times 10^{7} \mathrm{~mm}^{-1} \\
b=1.05\end{array}$ & $\begin{array}{l}\text { Set 4. Dislocation Density } \\
\text { Static Thermal Recovery } \\
\mathrm{C}_{\rho \mathrm{R}}=c_{R} \exp \left(-\frac{\mathrm{Q}_{\text {self }}}{\mathrm{RT}}\right)^{k} \\
\text { where } c_{R}=1.30 \times 10^{7} \\
k=0.33\end{array}$ \\
\hline \begin{tabular}{|l|} 
Set 5. Back Stress \\
Static Thermal Recovery \\
$\beta=A_{2} \exp \left(\frac{Q_{\text {self }}}{\mathrm{RT}}\right)^{m}$ \\
where $\mathrm{Q}_{\text {self }}=280 \mathrm{~kJ} / \mathrm{mol}$ \\
$\mathrm{A}_{2}=121$ \\
$m=0.5$ \\
$m$
\end{tabular} & $\begin{array}{l}\text { Set 6. Microtwinning } \\
\text { Diffusion Coefficient } \\
\mathrm{D}_{\text {ord }}=\mathrm{D}_{0} \exp \left(-\frac{\mathrm{Q}_{\text {diff }}}{\mathrm{RT}}\right) \\
\text { where } \mathrm{Q}_{\text {diff }}=280 \mathrm{~kJ} / \mathrm{mol} \\
\mathrm{D}_{0}=3.39 \times 10^{-5}\end{array}$ \\
\hline
\end{tabular}

Table II. Calibrated temperature dependent parameter values at $706^{\circ} \mathrm{C}$ and $760^{\circ} \mathrm{C}$

\begin{tabular}{|c|l|c|c|}
\hline Set \# & \multicolumn{1}{|c|}{ Temperature } & $706^{\circ} \mathrm{C}$ & $760^{\circ} \mathrm{C}$ \\
\hline \hline \multirow{3}{*}{1} & $\mathrm{c}_{\mathrm{p} 2 \mathrm{~L}}$ & 0.0887 & 0.873 \\
\cline { 2 - 4 } & $\mathrm{c}_{\mathrm{p} 2 \mathrm{~S}}$ & 4.433 & 4.366 \\
\cline { 2 - 4 } & $\mathrm{c}_{\mathrm{p} 3}$ & $4.00 \times 10^{4}$ & $3.94 \times 10^{4}$ \\
\cline { 2 - 4 } & $\mathrm{c}_{\mathrm{gr}}\left(\mathrm{MPa} \cdot \mathrm{mm}^{-1 / 2}\right)$ & 6.19 & 6.10 \\
\hline \multirow{2}{*}{2} & $\mathrm{~h}_{\mathrm{pe}}$ & 0.44 & 0.24 \\
\cline { 2 - 4 } & $\mathrm{h}_{\mathrm{se}}$ & 0.22 & 0.12 \\
\hline 3 & $\mathrm{k}_{2}\left(\mathrm{~mm}^{-1}\right)$ & $3.06 \times 10^{-3}$ & 0.0100 \\
\hline 4 & $\beta$ & $3.7 \times 10^{-6}$ & $4.0 \times 10^{-6}$ \\
\hline 5 & $\mathrm{C}_{\rho \mathrm{R}}(\mathrm{MPa})$ & 50.5 & 100.0 \\
\hline 6 & $\mathrm{D}_{\text {ord }}\left(\mathrm{m}^{2} / \mathrm{s}\right)$ & $3.7 \times 10^{-20}$ & $7.0 \times 10^{-19}$ \\
\hline
\end{tabular}




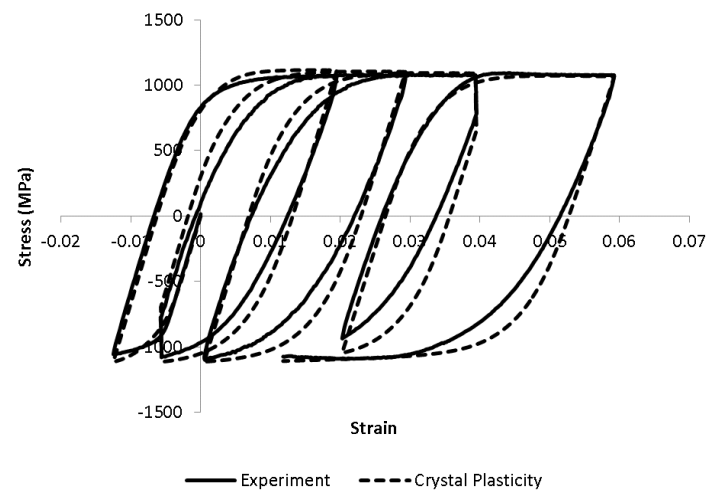

Figure 1. Example of calibrated crystal plasticity model response compared to experimental data with $\mathrm{M} 2$ microstructure at $760^{\circ} \mathrm{C}$ and $10^{-5} \mathrm{~s}^{-1}$

An additional deformation mechanism addressed by the crystal plasticity model is the microtwinning-governed deformation under creep. For calibrating microtwinning associated parameters, stress level sensitivity is considered along with temperature and microstructure sensitivities. The calibration process is conducted independently from that associated with strain-controlled experimental responses involving substantial cyclic plasticity, as it is essentially decoupled.

The microtwinning evolution is calibrated to the 11 experimental creep responses $\left(10\right.$ at $706^{\circ} \mathrm{C}$ and 1 at $760^{\circ} \mathrm{C}$ ). At $706^{\circ} \mathrm{C}$, two microstructures are experimented to stress levels ranging from $655 \mathrm{MPa}$ to $793 \mathrm{MPa}$ with an increment of $34.5 \mathrm{MPa}$. The creep experiments demonstrate strong microstructure and stress level dependences.

As in the strain-controlled cases, microtwinning parameters are first individually calibrated to experimental responses at each stress level. Then constants in the microtwinning density evolution equation are calibrated to reflect the stress level sensitivity. Afterwards, the model is further calibrated to capture the microstructure sensitivity which is observed to be relatively significant in creep responses compared to strain-controlled responses. The microstructure sensitivity is implemented through coefficients associated with $\gamma^{\prime}$ particle sizes and channel widths which affect both microtwinning velocity and density. The temperature effect, however, is only tentatively embedded as there is only one creep experiment conducted at $760^{\circ} \mathrm{C}$. Therefore, the temperature sensitivity obtained from one microstructure is ascribed to the other microstructure without further validation. The calibrated model is capable of reasonably simulating creep responses, with a variation of accuracy among histories. Model predictions at $655 \mathrm{MPa}$ and $793 \mathrm{MPa}$ with M1 microstructure at $706^{\circ} \mathrm{C}$ are compared to the experimental creep response in Figure 2.

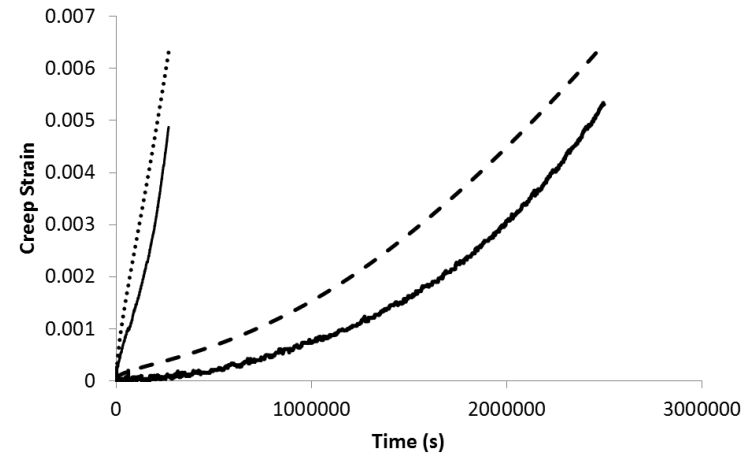

$$
\begin{array}{ll}
\text {..... } 793 \mathrm{MPa} \text { Model } & -793 \mathrm{MPa} \text { Experiment } \\
\text { - }-655 \mathrm{MPa} \text { Model } & -655 \mathrm{MPa} \text { Experiment }
\end{array}
$$

Figure 2. Calibrated crystal plasticity model response compared to the experimental creep response at $706^{\circ} \mathrm{C}$ with $655 \mathrm{MPa}$ and 793 $\mathrm{MPa}$ applied stress with M1 microstructure.

It should be noted that it is relatively simple to calibrate a constitutive relation to a single experiment, whereas it is extremely difficult to capture multiple sensitivities and macroscopic material behavior simultaneously and sufficiently over many experimental histories corresponding to various loading conditions. Therefore, a certain degree of compromise in the performance of the constitutive relation on a part of experimental conditions is unavoidable. Table III lists calibrated constants that are independent of microstructure and temperature considered in this study.

Table III. Calibrated crystal plasticity model parameters that have no temperature dependence

\begin{tabular}{|l|c||l|c|}
\hline$\dot{\gamma}_{1}\left(\mathrm{~s}^{-1}\right)$ & $6.1 \times 10^{16}$ & $\mathrm{D}_{\text {oct }}^{\alpha}(\mathrm{MPa})$ & 150 \\
\hline $\mathrm{n}_{1}$ & 5.0 & $\mathrm{D}_{\text {cub }}^{\alpha}(\mathrm{MPa})$ & 180 \\
\hline $\mathrm{h}_{\lambda}^{\alpha \alpha}$ & 2.0 & $\alpha_{\mathrm{t}}$ & 0.1 \\
\hline $\mathrm{k}_{1}\left(\mathrm{~mm}^{-1}\right)$ & $2.5 \times 10^{6}$ & $\mathrm{C}$ & 0.7 \\
\hline $\mathrm{k}_{\delta}(\mathrm{mm})$ & $2.5 \times 10^{-3}$ & $\tau_{\text {o,oct }}^{\alpha}(\mathrm{MPa})$ & 32 \\
\hline $\mathrm{C}_{\chi}$ & 1000.0 & $\tau_{\text {o,cub }}^{\alpha}(\mathrm{MPa})$ & 64 \\
\hline$\eta_{\mathrm{o}}$ & 0.18 & $\mathrm{~b}_{\text {tp }}(\mathrm{nm})$ & 0.145 \\
\hline$\mu_{\gamma^{\prime}}(\mathrm{MPa})$ & 164034 & $\mathrm{~b}_{\text {full }}(\mathrm{nm})$ & 0.251 \\
\hline$\mu_{\gamma}(\mathrm{MPa})$ & 102738 & $\Gamma_{\mathrm{pt}}\left(\mathrm{Jm}^{-2}\right)$ & $7.0 \times 10^{-7}$ \\
\hline $\mathrm{b}_{\gamma}(\mathrm{nm})$ & 0.407 & $\Gamma_{\text {tt }}\left(\mathrm{Jm}^{-2}\right)$ & $2.0 \times 10^{-7}$ \\
\hline $\mathrm{b}_{\gamma^{\prime}}(\mathrm{nm})$ & 0.253 & $\mathrm{~m}$ & 13.0 \\
\hline
\end{tabular}

\section{Application}

The calibrated crystal plasticity model can be used to analyze material behavior for microstructures in the range of those used for calibration and may be useful in supporting parametric study to design microstructures. 


\section{Conclusion}

In this work, a grain scale crystal plasticity constitutive model has been developed that includes microtwin nucleation and growth to augment octahedral and cube slip relations. The model is formulated based on phase field model simulation results and previous fine scale analyses. The model is further validated and calibrated using experimental responses of polycrystalline specimens subjected to various complex loading histories conducted with two microstructures at $704^{\circ} \mathrm{C}$ and $760^{\circ} \mathrm{C}$. The calibrated crystal plasticity model can simulate macroscopic deformation under a reasonably wide range of loading conditions and microstructures.

The model has shown the potential to reflect two distinct deformation mechanisms, microtwinning and slip, while sensitivities in strain rate, temperature, dwell periods and microstructure are sufficiently captured. The crystal plasticity model can be further improved and refined when more detailed microstructure relations relative to microtwin growth are investigated. Additionally, a wider range of microstructures, possibly even with different Ni-base superalloys, can be employed to extend the applicability of the crystal plasticity model.

\section{Acknowledgements}

The authors are grateful for the support of the US Air Forcesponsored Metals Affordability Initiative (MAI) project entitled "Durable High Temperature Disk Material", on subcontract from Pratt \& Whitney (Paul Reynolds, Technical Monitor). Team members included Pratt \& Whitney, GE Aviation, the Georgia Institute of Technology, The Ohio State University and the University of Rhode Island.

\section{References}

1. M. Shenoy et al., "Microstructure-sensitive modeling of polycrystalline IN 100," International Journal of Plasticity, 24 (10) (2008), 1694-1730.

2. R.C. Reed, The superalloys: fundamentals and applications. (Cambridge:Cambridge University Press., 2006).

3. S. Karthikeyan et al., "Modeling microtwinning during creep in Ni-based superalloys," Scripta Materialia, 54 (6) (2006), 11571162 .

4. R.R. Unocic et al., "Mechanisms of creep deformation in polycrystalline Ni-base disk superalloys," Materials Science and Engineering A, 483-484 (1-2C) (2008), 25-32.

5. R.R. Unocic et al., "Dislocation decorrelation and relationship to deformation microtwins during creep of a $\gamma^{\prime}$ precipitate strengthened Ni-based superalloy," Acta Materialia, 59 (19) (2011), 7325-7339.

6. G.B. Viswanathan et al., "Microtwinning during intermediate temperature creep of polycrystalline Ni-based superalloys: mechanisms and modelling," Philosophical Magazine,, Vol. 86 (Nos. 29-31) (2006), 4823-4840.

7. M. Shenoy, "Constitutive modeling and life prediction in nickel-base superalloys" (Ph.D. Thesis, Georgia Institute of Technology, 2006).

8. C.P. Przybyla, D.L. McDowell, "Microstructure-sensitive extreme value probabilities for high cycle fatigue of Ni-base superalloy IN100," International Journal of Plasticity, 26 (3) (2010), 372-394.
9. L. Kovarik et al., "Microtwinning and other shearing mechanisms at intermediate temperatures in Ni-based superalloys," Progress in Materials Science, 54 (6) (2009), 839873.

10. C. Shen et al., "Phase Field Modeling of Microstructural Evolution in Solids: Effect of Coupling among Different Extended Defects," Metallurgical and Materials Transactions A, 39 (7) (2008), 1630-1637.

11. M.Y. Sheh, D.C. Stouffer, "A crystallographic model for the tensile and fatigue response for Rene N4 at 982C," Transactions of the ASME. Journal of Applied Mechanics, 57 (1) (1990), 25-31. 12. V. Paidar et al., "A theory of the anomalous yield behavior in L12 ordered alloys," Acta Metallurgica, 32 (3) (1984), 435-448.

13. J.C. Moosbrugger, D.L. McDowell, On a class of kinematic hardening rules for nonproportional cyclic plasticity. In Chicago, IL, USA, Publ by American Soc of Mechanical Engineers (ASME), 1988, 79-89.

14. X. Feaugas, H. Haddou, "Grain-size effects on tensile behavior of nickel and AlSl 316L stainless steel," Metallurgical and Materials Transactions A: Physical Metallurgy and Materials Science, 34 A (10) (2003), 2329-2340.

15. A.S. Khan, S. Huang, Continuum theory of plasticity. (New York, NY:John Wiley \& Sons, Inc., 1995).

16. G.B. Viswanathan et al., "Investigation of creep deformation mechanisms at intermediate temperatures in René 88DT," Acta Materialia, 53 (10) (2005), 3041-3057.

17. L. Kovarik et al., "The intermediate temperature deformation of Ni-based superalloys: Importance of reordering," JOM, 61 (2) (2009), 42-48.

18. R.R. Unocic et al., Deformation mechanisms in ni-base disk superalloys at higher temperatures. In 11th International Symposium on Superalloys, Superalloys 2008, September 14, 2008 - September 18, 2008. Champion, PA, United states, Minerals, Metals and Materials Society, 2008, 377-385.

19. M. Kolbe, "The high temperature decrease of the critical resolved shear stress in nickel-base superalloys," Materials Science and Engineering A, 319-321 (2001), 383-387.

20. ABAQUS (2006) Version 6.7, Simulia Inc., Providence, RI. 21. R.D. McGinty, "Multiscale representation of polycrystalline inelasticity" (Ph.D. Thesis, Georgia Institute of Technology, 2001). 